\title{
Fractal scaling of apparent soil moisture estimated from vertical planes of Vertisol pit images
}

\author{
Ramiro Cumbrera ${ }^{\mathrm{a}}$, Ana M. Tarquis ${ }^{\mathrm{b}}$, Gabriel Gascó ${ }^{\mathrm{c}}$, Humberto Millán ${ }^{\mathrm{a}, *}$ \\ ${ }^{a}$ Department of Basic Sciences, University of Granma, Apdo. 21, Bayamo, 85100 Granma, Cuba \\ ${ }^{\mathrm{b}}$ CEIGRAM, ETS Ing. Agrónomos (ETSIA), Universidad Politécnica de Madrid (UPM), Ciudad Universitaria, s.n., 28040 Madrid, Spain \\ ${ }^{c}$ Departamento de Edafología, ETS Ing. Agrónomos (ETSIA), Universidad Politécnica de Madrid (UPM), Ciudad Universitaria, s.n., 28040 Madrid, Spain
}

\section{S U M M A R Y}

Image analysis could be a useful tool for investigating the spatial patterns of apparent soil moisture at multiple resolutions. The objectives of the present work were (i) to define apparent soil moisture patterns from vertical planes of Vertisol pit images and (ii) to describe the scaling of apparent soil moisture distribution using fractal parameters. Twelve soil pits $(0.70 \mathrm{~m}$ long $\times 0.60 \mathrm{~m}$ width $\times 0.30 \mathrm{~m}$ depth $)$ were excavated on a bare Mazic Pellic Vertisol. Six of them were excavated in April/2011 and six pits were established in May/2011 after 3 days of a moderate rainfall event. Digital photographs were taken from each Vertisol pit using a Kodak ${ }^{\mathrm{TM}}$ digital camera. The mean image size was $1600 \times 945$ pixels with one physical pixel $\approx 373 \mu \mathrm{m}$ of the photographed soil pit. Each soil image was analyzed using two fractal scaling exponents, box counting (capacity) dimension $\left(D_{B C}\right)$ and interface fractal dimension $\left(D_{i}\right)$, and three prefractal scaling coefficients, the total number of boxes intercepting the foreground pattern at a unit scale $(A)$, fractal lacunarity at the unit scale $\left(A_{1}\right)$ and Shannon entropy at the unit scale $\left(S_{1}\right)$. All the scaling parameters identified significant differences between both sets of spatial patterns. Fractal lacunarity was the best discriminator between apparent soil moisture patterns. Soil image interpretation with fractal exponents and prefractal coefficients can be incorporated within a site-specific agriculture toolbox. While fractal exponents convey information on space filling characteristics of the pattern, prefractal coefficients represent the investigated soil property as seen through a higher resolution microscope. In spite of some computational and practical limitations, image analysis of apparent soil moisture patterns could be used in connection with traditional soil moisture sampling, which always renders punctual estimates.

\section{Introduction}

Image analysis is a modern tool for quantifying the morphology of spatial patterns of soil properties. Many works have been conducted in this direction using both, 2D and 3D gray-level or binary (e.g. black and white) images. In particular, image analysis seems to be a useful tool for describing vegetative developmental stages (Behrens and Diepenbrock, 2006), texture recognition (Kilic and Abiyev, 2011) or quantification of differential growth processes in plant root and shoot growth zones (Chavarría-Krauser et al., 2007). Combinations of image and fractal analyses have been used for characterizing bulk density patterns (Zeng et al., 1996), soil macroporosity (Gantzer and Anderson, 2002), soil micromorphology (Bartoli et al., 2005) and soil structural state (Dathe and Thullner, 2005). In almost all previously cited studies, the box counting (capacity) dimension has been considered as the main parameter for characterizing the scaling behavior of the investigated soil property. Some recent works have also used multifractal measures for characterizing water fingering from magnetic resonance images (Posadas et al., 2009) and mass and entropy dimensions derived from 3-D soil images (Tarquis et al., 2008). In general, some other indices as fractal lacunarity and entropy scaling need to be incorporated as complementary parameters. Some studies have used fractal lacunarity for landscape texture evaluation (e.g. Plotnick et al., 1993) or scale-dependent clustering of fracture networks (Roy et al., 2010), but it is still a less considered scaling parameter. Even though most hydraulic soil properties are direct or indirect consequences of soil moisture distribution, there are relatively few works using 2-D or 3-D image analysis for describing soil moisture scaling. Due to the evident visual contrast among wetter and drier zones, image and fractal analyses can be useful for characterizing the local and global distribution of soil moisture within soil profiles. The objectives of the present work were (i) to define apparent soil moisture patterns from vertical planes of Vertisol pit images and (ii) to describe the scaling of apparent soil moisture distribution using fractal parameters. 


\section{Theoretical considerations}

Here we present a brief overview of the main fractal and prefractal parameters involved in the present work and the way they have been estimated.

\subsection{Box counting (capacity) dimension}

The use of box counting rationale for computing fractal (capacity) dimension assumes that the investigated pattern (e.g. soil moisture) fits some or all of the strong symmetries (e.g. translational, rotational and/or dilation invariance) (Feder, 1988) which can be called scaling invariance. For a 2-dimensional image one has to divide a Euclidean box of linear size, $L$, which contains the pattern, into $(L / r)^{2}$ smaller boxes each of linear size $r$. The number of non-overlapping boxes, $N_{i}$, of size $r_{i} \leqslant L$ containing pieces of the pattern (for example wet pixels) follows a power law:

$N_{i}\left(L, r_{i}\right) \propto\left(\frac{L}{r_{i}}\right)^{D_{B C}}$

where $D_{B C}$ is the box counting or fractal capacity dimension. In practice, Eq. (1) represents a limit as $r_{i} \rightarrow 0$, which imposes some restrictions. For example, $L$ represents the finite system size condition (upper cutoff) while always $r_{i} \neq 0$ (Baveye et al., 2008).

Eq. (1) was used with experimental data in the form:

$N(r)=A r^{-D_{B C}}$

where $A$ is a scaling coefficient accounting for the number of boxes intercepting the considered pattern (e.g. wet or dry zones) at the unit scale (e.g. $r \rightarrow 1$ ). One could note from Eq. (1) that:

$A \propto L^{D_{B C}}$

which connects the $A$ coefficient directly with the fractal scaling of the investigated soil property or image pattern $\left(D_{B C}\right.$ in this case $)$ and the initiator size, L. A log-log transformation of Eq. (2) allows one to estimate $D_{B C}$ as the slope of the linear regression equation and $\log (A)$ as the corresponding intercept. That is:

$\log (N)=\log (A)-D_{B C} \log (r)$

To our knowledge, only Kravchenko et al. (2011) and few other workers have paid attention to the potential utility of the A coefficient as another scaling constant. Thus, within the context of the present work the A coefficient is used for estimating a corrected value of the apparent soil moisture.

\subsection{Interface fractal dimension}

Interfaces are geometrical structures separating two or more phases in soil system (e.g. pore-solid or dry-wet interface). From a theoretical point of view, a real world interface mimics, to some extent, the random counterpart of the deterministic von Koch curve. Many important physical, chemical and biological phenomena occur just at those boundaries. In principle, the box counting fractal theory is appropriate for estimating the complex geometry of such boundaries. Thus, Eqs. (1), (2), and (4) are valid for a quantitative description of such irregular interfaces. However, in this case $D_{B C}=D_{i}$ (the interface fractal dimension) and $N=N_{s}(r)$ is the number of boxes covering the interface at each resolution, $r$. For the case of the dry-wet frontier, the $N_{s}(r)$ value can be calculated using the same equation in Dathe and Thullner (2005):

$N_{s}(r)=N_{w}(r)+N_{d}(r)-N_{\max }(r)$

where $N_{w}$ and $N_{d}$ are the number of boxes covering wet and dry zones, respectively, and $N_{\max }$ is the total number of boxes covering the entire image at each resolution, $r$. In particular, $N_{\max }(r)$ can be calculated using a simple equation:

$N_{\max }(r)=\frac{N_{p}}{r_{i}^{2}}, \mathrm{r}_{\mathrm{i}}=1,2,4, \ldots, \mathrm{L}$

where $N_{p}$ is the total number of pixels covering the image.

\subsection{Fractal lacunarity}

Fractal lacunarity is a complementary measure for objects with similar fractal dimensions (Mandelbrot, 1983; Kaye, 1989). Allain and Cloitre (1991) defined lacunarity as a scale-dependent measure of heterogeneity of an object, whether or not it is fractal. In other words, it is the deviation of a fractal object from translational invariance. Briefly, lacunarity conveys information on the density of occupation of massless zones within the fractal object. For the sake of completeness, the term succolation is also complementary to lacunarity for fractal systems where percolation can occur (e.g. soils). According to Mandelbrot (1977) definition, a succolating system is one close to percolation. The theoretical background for computing lacunarity using the gliding box method is reported in many papers (e.g. Allain and Cloitre, 1991; Plotnick et al., 1993; Baveye et al., 2008; Przemyslaw, 2009). We reproduce it briefly within the context of binary images.

A box of size $r$ is positioned at the origin of the binary image. As the box moves (e.g. moving window) through the image, it is calculated the number, $n$, of black pixels within the box at each position (let us assign a "mass", $m$, to this box). This procedure renders a frequency distribution function $n(m, r)$ which is converted into a probability distribution function $P(m, r)$ after dividing by the total number of boxes $N(r)$ of size $r$. Now, the first $\left(Q_{1}\right)$ and second $\left(Q_{2}\right)$ order statistics of the distribution can be determined as:

$Q(1)=\sum m P(m, r)$

$Q(2)=\sum m^{2} P(m, r)$

The lacunarity for the specific box size was computed as:

$\Lambda(r)=1+\frac{\sum m^{2} P(m, r)}{\left[\sum m P(m, r)\right]^{2}}$

Note that for a non-lacunar structure (e.g. translationally invariant) $\Lambda(r)=1$, which suggests that lacunarity is statistically a measure of the distribution width (Baveye et al., 2008).

An interesting question refers to the scaling of $\Lambda(r)$ as a Pareto law of $r$.

$A(r)=A_{1} r^{-b}$

Here we interpret $A_{1}$ as the lacunarity at a unit scale while $b$ is a scaling exponent. Both, $A_{1}$ and $b$ can be estimated from the loglog transformation:

$\log A(r)=\log \Lambda_{1}-b \log r$

\subsection{Scale-dependent Shannon entropy}

The distribution of white/black pixels within a 2-dimensional soil image can be very heterogeneous. In fact, Shannon entropy might be an interesting informational measure of the effective measure of the investigated distribution. Here, one takes the advantage that a soil image can be partitioned into several boxes of sizes $r_{1}, r_{2}, \ldots, r_{n}$.

For discrete distributions, the representation of the Shannon entropy as a function of the box size, $r$, is:

$S(r)=-\sum_{i=1}^{n} p_{i}(r) \log p_{i}(r)$ 
Within the context of the present work, $p_{i}(r)$ represents the probability that a given mass of the image (e.g. white pixels) is in the box of size $r$. That is:

$p_{i}(r)=\frac{N_{w}(r)}{N_{\max }(r)}$

where $N_{w}(r)$ is the number of white pixels within the box of size $r$ and $N_{\max }(r)$ is the total number of pixels (e.g. white + black pixels) within the same box. From Eq. (12) one can define the entropy or information dimension $\left(D_{E}\right)$ as:

$D_{E}=\lim _{r \rightarrow 0} \frac{S(r)}{\log \left(\frac{1}{r}\right)}$

Martin and Taguas (1998) have used the $D_{E}$ index for classifying soil textures.

From a practical point of view, one can use a log-log transformation of Eq. (14):

$S(r)=S_{1}-D_{E} \log (r)$

In this case, $S_{1}$ is the entropy at the unit scale.

In the present work we paid special attention to all the scaling prefactors $\left(A, \Lambda_{1}\right.$ and $S_{1}$ ). Note that as $r \rightarrow 1$, one obtains the maximum number of boxes paving the image.

\section{Materials and methods}

\subsection{Soil characteristics and sampling}

The study site is located in Veguitas, Cuba $\left(76^{\circ} 54^{\prime} \mathrm{W}, 20^{\circ} 16^{\prime} \mathrm{N}\right)$. This area belongs to Cauto river plain, which is the largest hydrographic basin in Cuba. The soil is classified as a Mazic Pellic Vertisol (World Reference Base for Soil Resources, 2006). Even though different crops and tillage treatments have been established during the last 40 years, at the time of the present study the soil was not in use. Fig. 1 shows an overview of the study site.

A total of twelve pits were considered in the present investigation. Six pits $(0.70 \mathrm{~m}$ long $\times 0.60 \mathrm{~m}$ width $\times 0.30 \mathrm{~m}$ depth) were excavated in April/2011 and six pits were established in May/ 2011 after 3 days of a moderate rainfall event. Each pit was separated approximately $1.5 \mathrm{~m}$ each other. Thus, we considered two different scenarios: before and after a rainfall event. Soil samples were also collected for conducting physical (e.g. soil texture, gravimetric water content) and chemical (e.g. pH, organic matter) analyses. In this case, we mixed soil samples collected from the six pits before the rainfall event. After rainfall, only gravimetric water

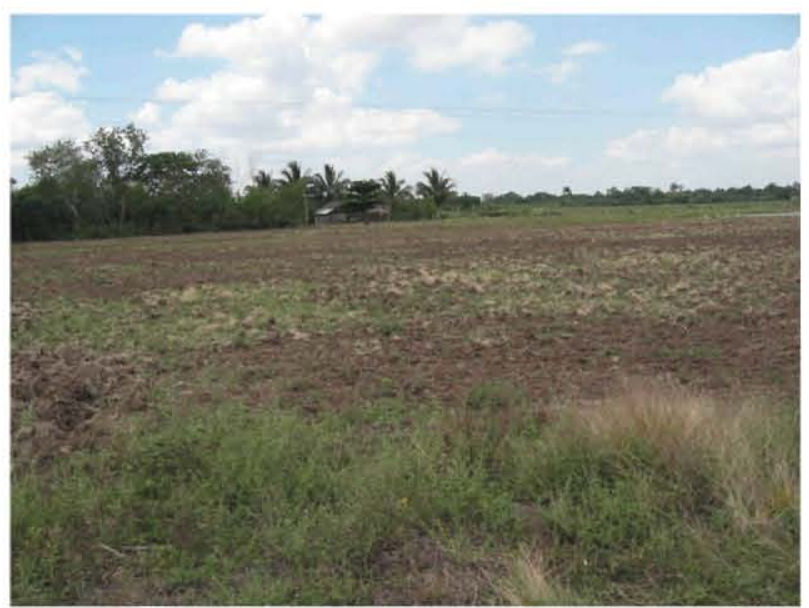

Fig. 1. Photograph showing the investigated field
Table 1

Some soil physical and chemical characteristics of the studied site.

\begin{tabular}{lll}
\hline Soil property & Before rainfall & After rainfall \\
\hline Clay (\%) & $54.8(6.2)$ & n.d \\
Silt (\%) & $31.6(5.5)$ & n.d \\
Sand (\%) & $13.6(6.3)$ & n.d \\
$\mathrm{pH}(-)$ & $7.2(0.25)$ & n.d \\
OM (\%) & $3.5(0.75)$ & n.d \\
$W\left(\mathrm{~kg} \mathrm{~kg}^{-1}\right)$ & $0.245(0.06)$ & $0.442(0.08)$ \\
\hline
\end{tabular}

${ }^{a}$ Not determined.

content was determined. Particle-size distributions were obtained using the pipette method (Gee and Bauder, 1986), soil pH was determined using the potentiometric method while organic carbon $(O C)$ was estimated by dry combustion (organic matter $=1.7240 C$ ). Gravimetric water content ( $W$ hereafter) was determined after oven-drying soil samples at $105^{\circ} \mathrm{C}$ during $24 \mathrm{~h}$. Each laboratory analysis was conducted using three replicates. Table 1 shows the main soil physical and chemical characteristics.

\subsection{Soil image processing}

Photographs were taken from each Vertisol pit using a Kodak ${ }^{\mathrm{TM}}$ digital camera (Kodak EasyShare C182, 12 Mp resolution). We considered only the pit wall with the better illumination and contrast. That is, we searched for a clear contrast between dark areas representing wet zones and light regions associated to dry areas. The mean total number of pixels of the digital images was $1,512,000$ pixels $(1600 \times 945$ pixels). That is, one pixel of the digital image corresponded, approximately, to $373 \mu \mathrm{m}$ of the photographed soil pit (e.g. one physical pixel $=0.0373 \mathrm{~cm}$ ). Some authors (e.g. Tanaka et al., 1999) have recommended larger images to avoid, to some extent, the influence of image size on fractal parameters.

The present work was developed under field conditions. A specific computational code (VertiSoft. 1.0, available upon request) was developed for processing soil images. This code runs under MATLAB $^{\circledR}$ environment (The MathWorks, Inc., 2005). We tested five different computational filters for smoothing soil images: $3 \times 3$, $5 \times 5,7 \times 7,9 \times 9$ and $11 \times 11$ mean filters. Each RGB matrix of the resulting image was segmented using multivariate $k$-means cluster analysis. In this case, we selected seven clusters for each RGB matrix. This procedure allows one to smooth those curves describing link distances between different values with regard to the original matrix. In addition, histograms were generated for checking the separation of gray scale intensities corresponding to the foreground (wet zones) from those intensities of the background (drier zones) (Glasbey and Horgan, 1995). The aforementioned methodology showed that $9 \times 9$ mean filter was the better choice for all the images. Fig. 2 illustrates the differences between the original RGB image and that processed with $9 \times 9$ mean filter for the particular case of pit no. 6 . After that, we extracted the characteristic moisture pattern using the algorithm of pixel detection. The final result was a binary image showing black (0) and white (1) zones representing apparent dry and wet areas, respectively.

\subsection{Fractal and prefractal scaling analysis}

Box counting $(N, r)$ and entropy $(S, r)$ values were computed using the Image J Software (Rasband, 2006) with each binary image, and resolutions (box sizes) 1, 2, 4, 8, 16, 32, 64, 128 and 256 pixels. That scaling range does not exceeded $30 \%$ of the image size as recommended by some authors (Dathe et al., 2001). As we were also interested in the parameter value at the unit scale, all the paired data were converted into excel worksheets and then imported 
(a) Means for each cluster (original image)

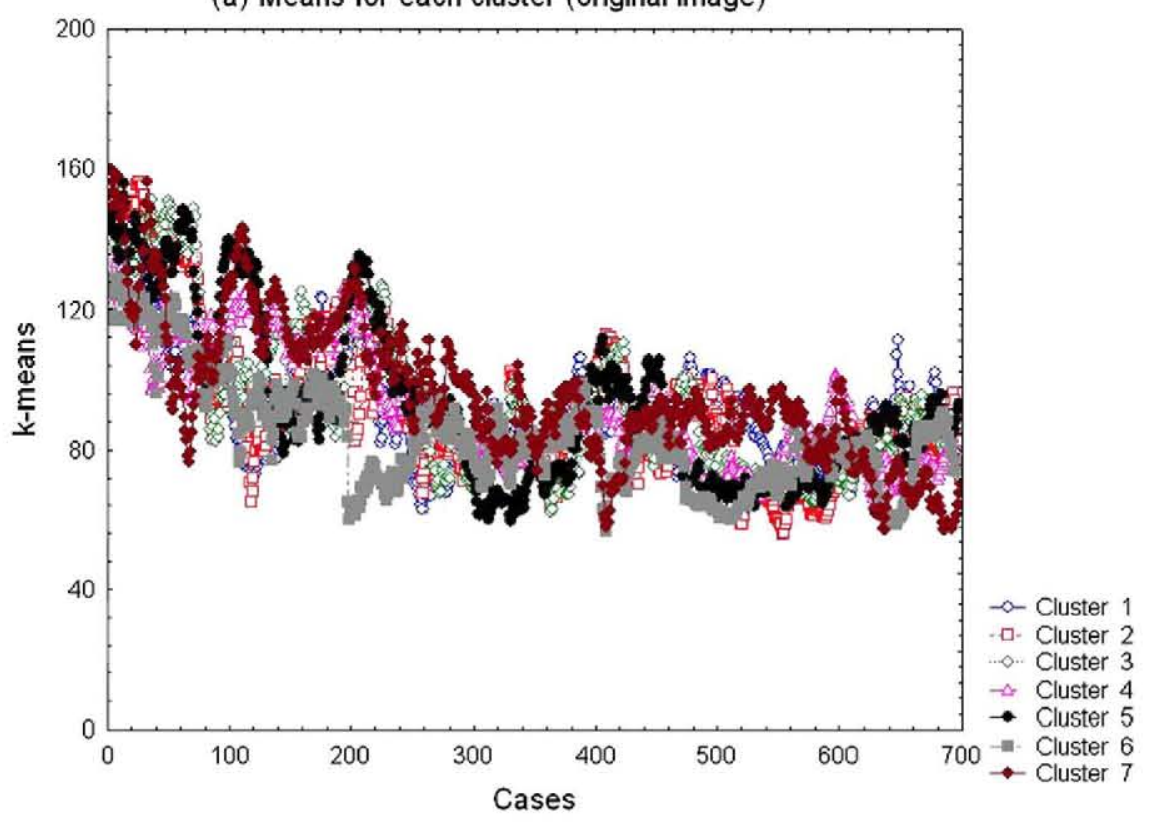

(b) Means for each cluster (filtered image)

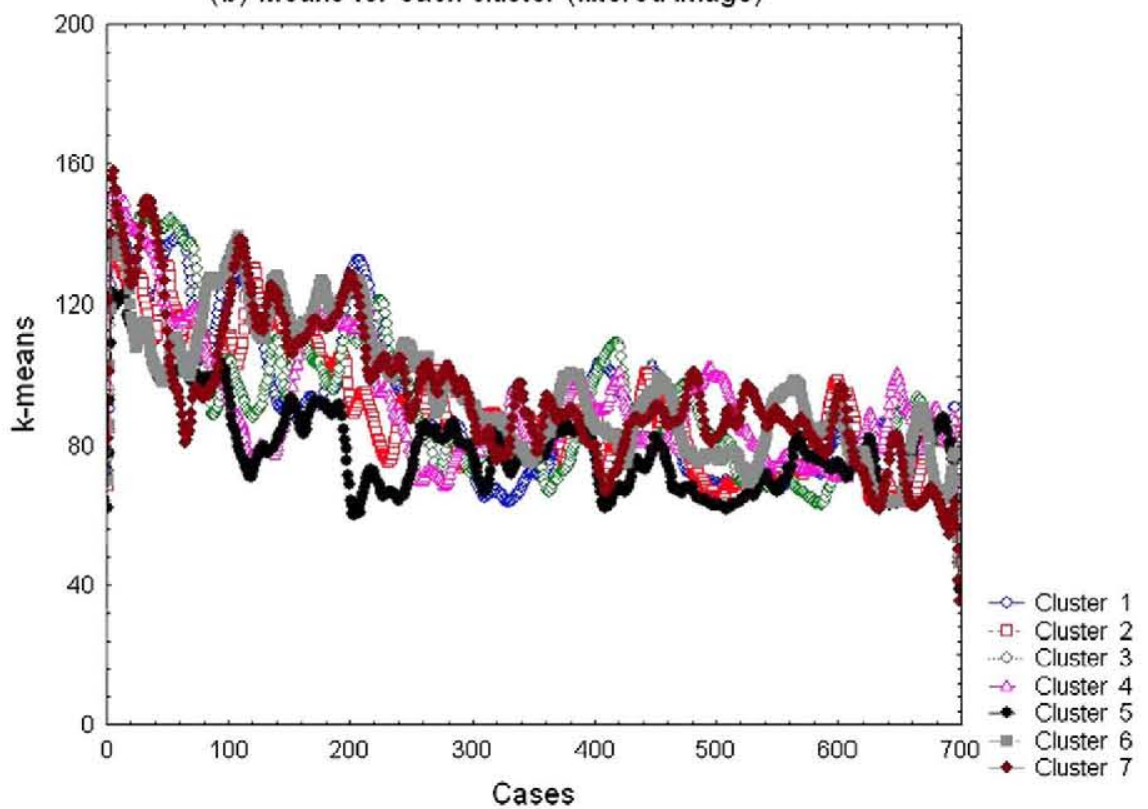

Fig. 2. Multivariate $k$-means clustering for pit no. 6 image before the rainfall event: (a) original RGB image, (b) $9 \times 9$ mean filter application.

from Statistica ${ }^{\mathrm{TM}}$ Software Package (StatSoft, Inc., 2007). In the present study white color represents wet zones (foreground) while black color corresponds to dry areas (background). Fractal lacunarity analysis was conducted using the FRACLAC Software (Karperien, 2007), which is a plugin for ImageJ (Rasband, 2006).

In the case of box counting data, we performed the analysis on both, white (object) and black (background) regions for calculating an apparent soil moisture $\left(\theta_{c}\right.$ hereafter). That is:

$\theta_{c}=\frac{N_{w}(r=1)}{N_{w}(r=1)+N_{b}(r=1)}$

where $N_{w}(r=1)$ is the total number of boxes of size $r=1$ pixel for white (object) zones and $N_{b}(r=1)$ corresponds to black (background) areas. Those calculated apparent soil moisture values $\left(\theta_{c}\right)$ can be corrected if one uses the intercept, $\log (A)$, after fitting Eq. (4). That is:
$\theta_{e}=\frac{A_{w}(r=1)}{A_{w}(r=1)+A_{b}(r=1)}$

where $\theta_{e}$ is the estimated apparent soil moisture from the fractal model, $A_{w}(r=1)$ is the total number of boxes of size $r=1$ pixel for white (object) zones and $A_{b}(r=1)$ corresponds to black (background) areas.

\subsection{Statistical analysis}

We performed linear regression analyses on Eqs. (4), (11), and (15) at $p<5 \%$. Both sets of fractal dimensions and prefractal coefficients (before and after rainfall) were also compared using a 2-tail $t$-Student statistics. The aforementioned analyses were performed using the Statistica ${ }^{\mathrm{TM}}$ Software Package (StatSoft, Inc., 2007). 


\section{Results and discussion}

\subsection{Soil image processing}

Fig. 3 illustrates the performance of soil image processing (soil pit no. 6). That is, the gray scale soil image (Fig. 3 left) and the corresponding binary image after the application of $9 \times 9$ filter (Fig. 3 right). Dark gray color corresponds to apparent soil moisture distribution (Fig. 3 left). The arrows show that structures representing holes were interpreted as non-wetted zones. Bi-modal histograms (Fig. 4) showed a clear separation of the gray scale intensities corresponding to the foreground (wet zones) and background (dry zones), which indicated a minimal influence of regions overlapping. Many authors (Glasbey and Horgan, 1995; Dathe et al., 2001; Baveye, 2002) have previously stressed the importance of this problem. One can note a threshold of approximately 77 at the gray scale level before (Fig. 4 left) and after (Fig. 4 right) the rainfall event. In each case the peak below the threshold corresponded to wet areas (foreground). A comparison with the Otsu thresholding method (Otsu, 1979) rendered approximately a similar value for the same image (threshold value $=78$ ). It is possible that predominant clay mineral (montmorillonite in this case) could also influence on gray scale soil image. In fact, montmorillonitic soils show darker zones where water retention is higher. From our point of view, this could aid image thresholding. A common practice has been to consider the infiltration of coloring solutions into the soil profile (Hatano and Booltink, 1992; Hatano et al., 1992). Obviously, that approach enhances the overall quality of the image and subsequent analysis. However, in soil image analysis one always has to face subjective questions as appropriate image resolution, correct thresholding, image finite size and scaling effects. Baveye et al. (1998) have investigated some of the aforementioned points.

\subsection{Scaling parameters of apparent soil moisture patterns}

Eq. (4) fitted quite well each $N(r)$ versus $r$ data set before and after the rainfall event. Determination coefficients, $R^{2}$, were all $>0.996$. Fig. 5 a illustrates the model performance for the particular case of soil pit no. 6 before the rainfall event. $D_{B C}$ ranged from 1.42 to $1.77\left(\Delta D_{B C}=0.35\right)$ before rainfall and from 1.73 to 1.78 $\left(\Delta D_{B C}=0.05\right)$ after the moderate precipitation event. Thus, the geometrical space filling characteristics of the apparent moisture pattern was less variable after the rainfall event. An interesting point is that $D_{B C}$ stated clear differences between both scenarios (Table 2). Thus, $D_{B C}$ could be a valuable parameter for monitoring the space filling of apparent moisture patterns through soil profile
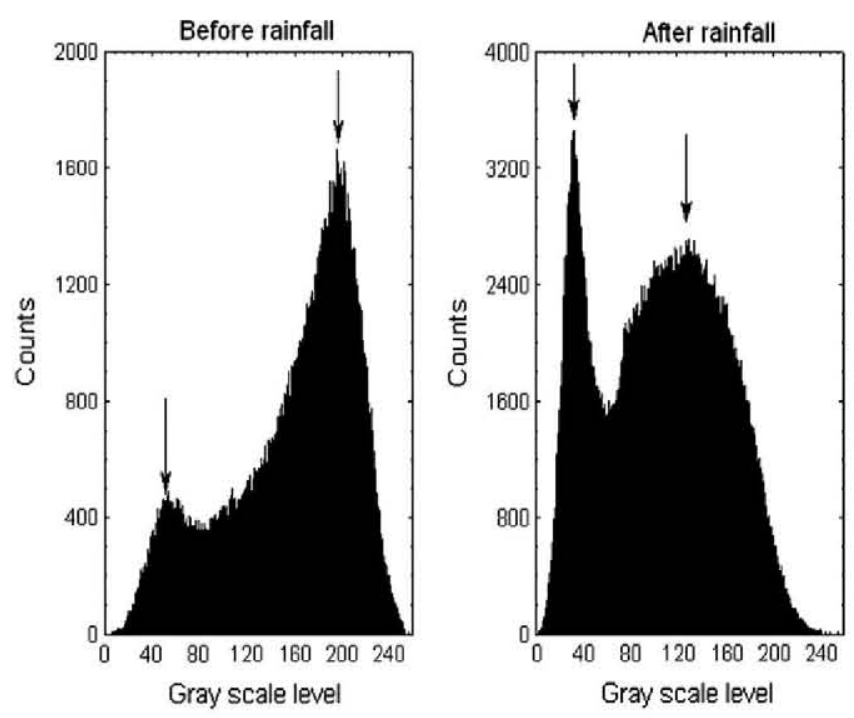

Fig. 4. Bi-modal histogram of gray scale levels (soil pit no. 6) before (left) and after the rainfall event (right).

before and after rainfall or irrigation. However, we recall here that $D_{B C}$ only could be insufficient for capturing the geometrical or morphological attributes of the spatial pattern. The aformentioned statement can be supported through the significant linear relationship between $D_{B C}$ and apparent moisture content as measured at a unit scale $\left(\theta_{e}\right)$ :

$\theta_{e}=0.932(0.08) \cdot D_{B C}+1.429(0.02), \mathrm{R}=0.963, \mathrm{n}=12$

At a first sight, Eqs. (16) and (17) look like the same thing. However, the results were different in approximately $2 \%$. That is, while $\theta_{c}$ was calculated from a counting procedure using only one observation scale (e.g. box size $=1$ pixel), $\theta_{e}$ was estimated from the lower cutoff of the fractal domain. Under that rationale, $\theta_{e}$ can be considered as another prefractal scaling parameter that allows one to correct over-estimations of the standard counting process. Note that $\theta_{e}$ also discriminated between the apparent soil moisture patterns before and after rainfall (Table 2 ).

Fig. 5b illustrates the performance of Eq. (4) with $N_{s}$ calculated from Eq. (5). In this case, the interface fractal dimension, $D_{i}$, ranged from 1.15 to $1.43\left(\Delta D_{i}=0.28\right)$ before rainfall and from 1.49 to 1.55 after precipitation $\left(\Delta D_{i}=0.06\right)$. We found significant differences between $D_{i}$ values before and after rainfall (Table 2). When one investigates apparent soil moisture patterns from image analysis, $D_{i}$ could be a useful parameter to gain information on the geometry
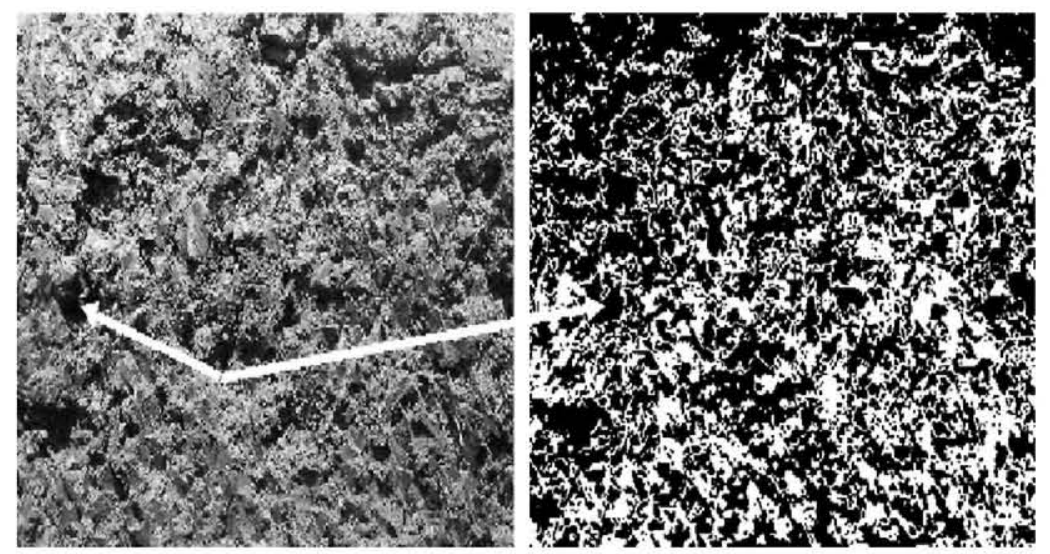

Fig. 3. Illustrative gray scale image (left) and its binary counterpart after filtering (right) (soil pit no. 6), 
(a) Box counting dimension

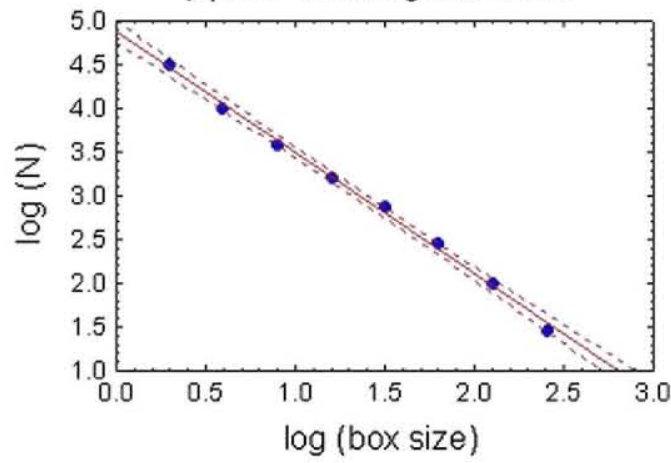

(c) Lacunarity scaling

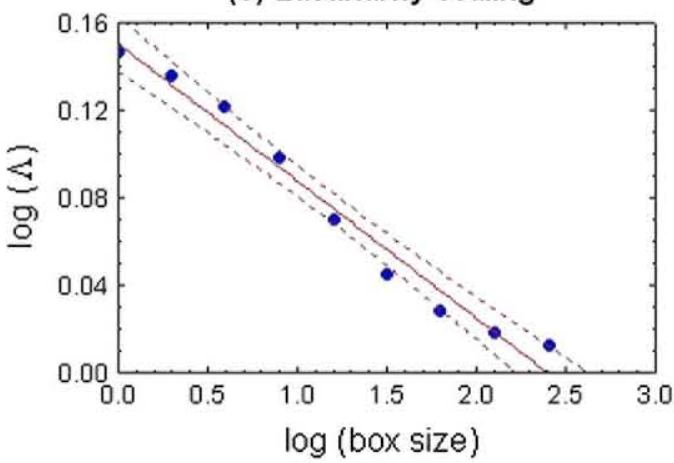

(b) Interface fractal dimension

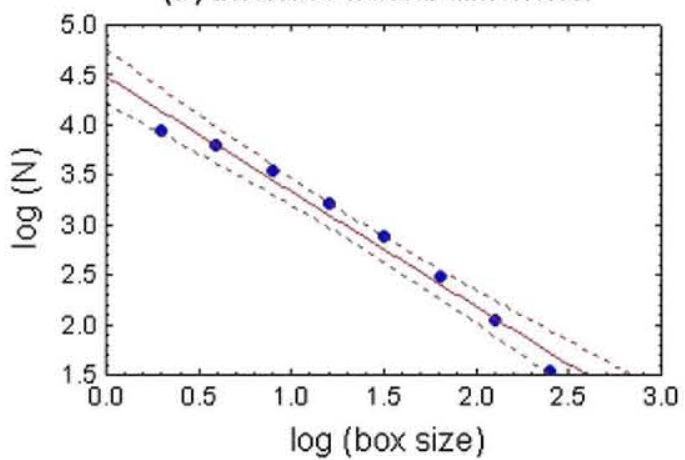

(d) Entropy scaling

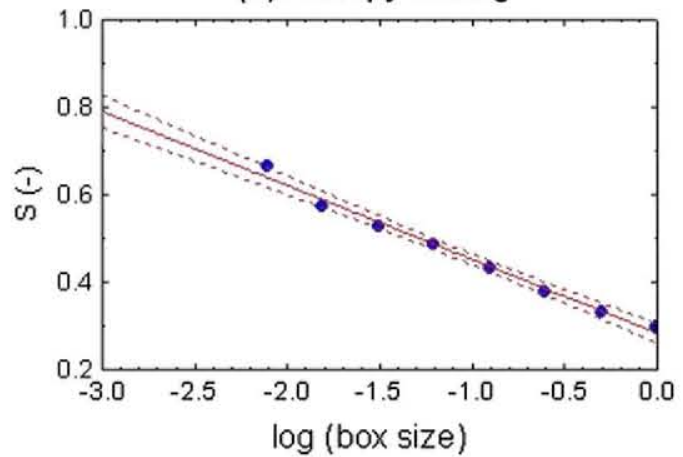

Fig. 5. Illustrative fitting of (a) Eq. (4), (b) Eq. (4) with Ns computed from Eq. (5), (c) Eq. (11) and (d) Eq. (15).

Table 2

Statistical comparison between both sets of parameters.

\begin{tabular}{lll}
\hline Parameter $^{*}$ & Before rainfall & Alter rainfall \\
\hline$\theta_{\mathrm{e}}$ (unit scale) (\%) & $18.21(11.8)^{\mathrm{b}}$ & $35.47(4.35)^{\mathrm{a}}$ \\
$D_{B C}{ }^{\mathrm{a}}$ & $1.59(0.11)^{\mathrm{b}}$ & $1.77(0.02)^{\mathrm{a}}$ \\
$A_{1}$ (unit scale) & $1.29(0.24)^{\mathrm{b}}$ & $1.62(0.16)^{\mathrm{a}}$ \\
$D_{i}$ & $1.31(0.09)^{\mathrm{b}}$ & $1.52(0.02)^{\mathrm{a}}$ \\
$S_{1}$ (unit scale) & $0.57(0.20)^{\mathrm{b}}$ & $0.89(0.05)^{\mathrm{a}}$ \\
\hline
\end{tabular}

Mean values ( \pm std.) in the same row followed by different lowercase letters differ statistically at $p<0.05$ according to a paired $t$-Student test.

${ }^{\text {a }} D_{B C}$ values correspond to white color (wetting pattern) in Fig. 3 (right).

and scaling of apparent soil moisture front, which in turns, could shed light on pore system occupation or vertical and lateral moisture displacement. For all soil images, $D_{i}$ had the smallest value in comparison with $D_{B C}$. This result agrees with previous studies conducted by Dathe and Thullner (2005). We found a significant linear relationship between $D_{i}$ and $\theta_{e}$ :

$$
\begin{aligned}
\theta_{e} & =0.905(0.11) \cdot D_{i}-1.01(0.16), R=0.925(p<0.05), n \\
& =12
\end{aligned}
$$

That is, as the fractal dimension of the dry-wet interface increased, the wetting front also increased. It is instructive to recall that $D_{i}$ represents a fractal, very complicated line of almost infinite length (Mandelbrot, 1967). Under that perspective, an increase of $D_{i}$ implies an increase of the fractal line separating wet from dry zones.

Fig. $5 \mathrm{c}$ shows the fitting of Eq. (11) (lacunarity curve) for the particular case of Vertisol pit no. 6 before rainfall. All the fits were excellent with determination coefficients $>0.975$. The fractal lacunarity at the unit scale, $\Lambda_{1}$, ranged from 1.083 to $1.774\left(\Delta \Lambda_{1}=\right.$ $0.691)$ before rainfall and from 1.412 to $1.901\left(\Delta \Lambda_{1}=0.489\right)$ after rainfall. Larger $A_{1}$ values after rainfall event means uneven distribution of the apparent soil moisture between different regions of the pattern. Such a greater degree of unoccupied sites clustering could explain, to some extent, the uneven growth of plant root system under field conditions. That information is difficult to extract from standard soil moisture sampling. Note that $\Lambda_{1}$ was a better discriminator between spatial patterns characteristics as compared to $D_{B C}$ or $D_{i}$. Based on Eq. (9), $A(r)$ was sensitive to the distribution of gaps that were not occupied by the apparent moisture pattern. In addition, $A(r)$ captured the scale-dependent statistics of the apparent soil moisture. Note also from Eq. (9) that $\Lambda(r) \rightarrow 1$ (second order statistics $\rightarrow 0$ ) as $r \rightarrow r_{\max }$, which supports, to some extent, the use of $\Lambda_{1}$ as a reference value. From our point of view, $\Lambda_{1}$ can provide useful information on micropore soil moisture invasion. In general, Plotnick et al. (1996) have stressed the potential usefulness of the application of fractal lacunarity analysis to quantitative and binary data. Furthermore, $\Lambda_{1}$ stated the most clear difference between apparent soil moisture patterns before and after precipitation (Table 2). It was found a significant linear relationship between $D_{B C}$ and the $b$ exponent estimated from Eq. (10):

$D_{B C}=1.46(0.02)+3.43(0.43) b, \mathrm{R}=0.931(\mathrm{p}<0.05), \mathrm{n}=12$

That is, as $b$ increased, $\Lambda(r)$ dropped (see Eq. (11) for instance) and $D_{B C}$ also increased as expected. Thus, Eq. (20) conveys the same information as Eq. (2) in Borys (2009). In addition, the particular case $b=0$ suggests that non-lacunar patterns can possess fractal structures, which is theoretically correct. We also found a linear relationship between the $b$ exponent (Eq. (10)) and $\theta_{e}$ :

$\theta_{e}=3.748(0.22) \cdot b+0.026(0.01), R=0.982$

Eq. (21) suggests a dependence between apparent moisture content at the unit scale (reference scale) and the fractal lacunarity scaling. That is, as $b$ increased ( $A$ dropped markedly as a function of $r$ ), $\theta_{e}$ also increased. Thus, under the scale-invariance assumption of pat- 
tern complexity, $\theta_{e}$ was replicated as the resolution decreased (e.g. larger box size values).

Fig. 5d shows a general overview of the Shannon entropy scaling as represented by Eq. (15). The entropy of white pixel distributions at the unit scale, $S_{1}$, ranged from 0.283 to 0.903 before rainfall $\left(\Delta S_{1}=0.62\right)$ and from 0.795 to $0.940\left(\Delta S_{1}=0.145\right)$ after precipitation ocurrence. This theoretical scaling parameter also detected significant differences between both groups of images (Table 2). The information dimension, $D_{E}$, ranged from 0.169 to 0.249 before $\left(\Delta D_{E}=0.08\right)$ and from 0.236 to $0.256\left(\Delta D_{E}=0.02\right)$ after rainfall. There were found significant differences between $D_{E}$ mean values before and after rainfall (results not shown). In general, Shannon (information) entropy could be also a useful parameter for evaluating the uncertainty associated to the probability density function (pdf) of white pixels. Based on the theoretical rationale provided by $\mathrm{Xu}$ (2006), one could assume that average lack of information after the rainfall event could be due to the larger number of boxes with linear size $r=1$ available for apparent moisture occupancy. On the contrary, smaller entropy values (e.g. before rainfall) could occur due to the smaller number of boxes required by the apparent moisture pattern. This information can be used for zooming the spatial pattern at specific resolutions (e.g. root size). From our point of view, interpretation of soil images through fractal and prefractal scaling parameters could be incorporated as another tool for a site-specific agriculture. While fractal exponents $\left(D_{B C}, D_{i}\right.$ and $\left.D_{E}\right)$ convey information on the space filling characteristics of some properties of the pattern (e.g. mass distribution, interface irregularity, information), those prefractal coefficients estimated at a unit scale $\left(A, A_{1}\right.$ and $\left.S_{1}\right)$ represented the investigated soil property as seen through a higher resolution microscope. Unfortunately, it is extremely difficult to know the real value of soil water content from images even though one can assume that gray scale contrast begins at a certain moisture threshold. In spite of that limitation, we consider that image analysis of apparent soil moisture patterns could be used in connection with traditional soil moisture sampling, which always renders punctual estimates based on small soil cores. In fact, standard methods for measuring soil moisture (e.g. gravimetric method) do not permit a detailed description of the wetting front, which is important for investigating unsaturated soil hydraulic conductivity. Image and fractal analyses can overcome that limitation.

\section{Conclusions}

We have used fractal and prefractal scaling with two sets of soil images collected at a field scale before and after a moderate rainfall event. The spatial patterns of apparent soil moisture were separated reasonably well without using any dying solution. Fractal exponents and prefractal coefficients of the Pareto-type laws were capable of differentiating between both sets of spatial patterns. However, fractal lacunarity was the better discriminator, which agrees well with many previous investigations. While box counting and interface dimensions accounted for the space filling characteristics of the spatial pattern, their corresponding prefractal coefficients allowed the estimation of the apparent soil property at the highest possible resolution. In particular, the interface fractal dimension could shed light on the extent and complexity of soil moisture front and its preferential pathway. We think image analysis could be incorporated within the toolbox of a site-specific agriculture.

\section{Acknowledgments}

We want to express our gratitude to the referees and journal editor for their time, suggestions and valuable critical comments on the original version of the manuscript. We are also grateful to several informatics undergraduate students who assisted us with the fieldwork and software design. We also acknowledge to the landowners for permitting the access to the investigated field. Second author acknowledges the funding provided by Spanish Ministerio de Ciencia e Innovación (MICINN) through Project No. AGL2010-21501/AGR.

\section{References}

Allain, C., Cloitre, M., 1991. Characterizing the lacunarity of random and deterministic fractal sets. Phys. Rev. A 44, 3552-3558.

Bartoli, F., Genevois-Gomendy, V., Royer, J.J., Niquet, S., Vivier, H., Grayson, R., 2005. A multiscale study of silty soil structure. Eur. J. Soil Sci. 56, 207-223.

Baveye, P., 2002. Comment on "Evaluation of biofilm image thresholding methods". Water Res. 36, 805-806.

Baveye, P., Boast, C.W., Ogawa, S., Parlange, J.-Y., Steenhuis, T., 1998. Influence of image resolution and thresholding on the apparent mass fractal characteristics of preferential flow patterns in field soils. Water Resour. Res. 34, 2783-2796.

Baveye, P., Boast, C.W. Gaspard, S., Tarquis, A.M., Millán, H., 2008. Introduction to fractal geometry, fragmentation processes and multifractal measures: theory and operational aspects of their application to natural systems. In: Senesi, N., Wilkinson, K.J. (Eds.), Biophysical Chemistry of Fractal Structures and Processes in Environmental Systems, IUPAC Series on Analytical and Physical Chemistry of Environmental Systems. John Wiley and Sons, Chichester, UK, pp. 11-67.

Behrens, T., Diepenbrock, W., 2006. Using digital image analysis to describe canopies of winter oilseed rape (Brassica napus L.) during vegetative developmental stages. J. Agron. Crop Sci. 192, 295-302.

Borys, P., 2009. On the relation between lacunarity and fractal dimension. Acta Phys. Polon. B 40, 1485-1490.

Chavarría-Krauser, A., Nagel, K.A., Palme, K., Schurr, U., Walter, A., Scharr, H., 2007. Spatio-temporal quantification of differential growth processes in root growth zones based on a novel combination of image sequence processing and refined concepts describing curvature production. New Phytol. 177, 811-821.

Dathe, A., Thullner, M., 2005. The relationship between fractal properties of solid matrix and pore space in porous media. Geoderma 129, 279-290.

Dathe, A., Eins, S., Niemeyer, J., Gerold, G., 2001. The surface fractal dimension of the soil-pore interface as measured by image analysis. Geoderma 103, 203-229.

Feder, J., 1988. Fractals. Plenum Press, New York, NY

Gantzer, C.J., Anderson, S.H., 2002. Computed tomographic measurement of macroporosity in chisel-disk and no-tillage seedbeds. Soil Till. Res. 64, 101-111.

Gee, G.W., Bauder, J.W., 1986. Particle-size analysis. In: Klute, A. (Ed.), Methods of Soil Analysis, 2nd ed. ASA, Madison, WI, pp. 383-411.

Glasbey, C.A., Horgan, G.W., 1995. Image Analysis for the Biological Sciences. Wiley, Chichester, United Kingdom.

Hatano, R., Booltink, H.W.G., 1992. Using fractal dimensions of stained flow patterns in a clay soil to predict bypass flow. J. Hydrol. 135, 121-131.

Hatano, R., Kawamura, N., Ikeda, J., Sauma, T., 1992. Evaluation of the effect of morphological features of flow paths on solute transport by using fractal dimensions of methylene blue staining patterns. Geoderma 53, 31-44.

Karperien, A., 2007. Fraclac for Imagej. Fraclac Advanced Useŕs Manual. Charles Sturt University, Australia, 38 pp.

Kaye, B.H., 1989. A Random Walk through Fractal Dimensions. VCH Publishers, New York, NY.

Kilic, K.I., Abiyev, R.H., 2011. Exploiting the synergy between fractal dimension and lacunarity for improved texture recognition. Signal Process. 91, 2332-2344.

Kravchenko, A.N., Wang, A.N.W., Smucker, A.J.M., Rivers, M.L., 2011. Long-term differences in tillage and land use affect intra-aggregate pore heterogeneity. Soil Sci. Soc. Am. J. 75, 1658-1666.

Mandelbrot, B.B., 1967. How long is the coast of Britain? Science 156, 636-638.

Mandelbrot, B.B., 1977. The Fractal Geometry of Nature. W.H. Freeman, San Francisco, CA.

Mandelbrot, B.B., 1983. The Fractal Geometry of Nature. W.H. Freeman Company, New York, NY.

Martin, M.A., Taguas, F.J., 1998. Fractal modelling, characterization and simulation of partile-size distribution in soil. Proc. Roy. Soc. Lond. A 454, 1457-1468.

Otsu, N., 1979. Threshold selection method from gray-level histograms. IEEE Trans. Syst. Man. Cybern. 9, 62-66.

Plotnick, R.E., Gardner, R.H., O'Neill, R.V., 1993. Lacunarity indices as measures of landscape texture. Landscape Ecology 8, 201-211.

Plotnick, R.E., Gardner, R.H., Hargrove, W.W., Prestegaard, K., Perlmutter, M., 1996. Lacunarity analysis: a general technique for the analysis of spatial patterns. Phys. Rev. E 53, 5461-5468.

Posadas, A., Quiroz, R., Tannús, A., Crestana, S., Vaz, C.M., 2009. Characterizing water fingering phenomena in soils using magnetic resonance imaging and multifractal theory. Nonlin. Processes Geophys. 16, 159-168.

Przemyslaw, B., 2009. On the relation between lacunarity and fractal dimension. Acta Phys. Polon. B 40, 1485-1490.

Rasband, W., 2006. ImageJ. Image Processing and Analysis in Java, version 1.410. National Institutes of Health, USA, $46 \mathrm{pp}$.

Roy, A., Perfect, E., Dunne, W.M., Odling, N., Kim, J.-W., 2010. Lacunarity analysis of fracture networks: evidence for scale-dependent clustering. J. Struct. Geol. 32, 1444-1449. 
StatSoft, Inc., 2007. STATISTICA (Data Analysis Software System), version 8.0, Tulsa, OK.

Tanaka, M., Kayama, A., Kato, R., Ito, Y., 1999. Estimation of the fractal dimension of fracture surface patterns by box-counting method. Fractals $7,335-340$.

Tarquis, A.M., Heck, R.J., Grau, J.B., Fabregat, J., Sánchez, M.E., Antón, J.M., 2008. influence of thresholding in mass and entropy dimension of 3-D soil images. Nonlin. Processes Geophys. 15, 881-891.

The MathWorks Inc., 2005. MATLAB(R): The Language of Technical Computing version 7.1. The MathWorks Inc., Natick, MA.
World Reference Base for Soil Resources, 2006. World Reference Base for Soil Resources. A framework for international classification, correlation and communication. World Soil Resources Reports No. 103. FAO, Rome, $145 \mathrm{pp}$.

$\mathrm{Xu}, \mathrm{Q}$, 2006. Measuring information content from observations for data assimilation: relative entropy versus Shannon entropy difference. Tellus 59A 198-209.

Zeng, Y., Gantzer, C.J., Peyton, R.L., Anderson, S.H., 1996. Fractal dimension and lacunarity of bulk density determined with $\mathrm{X}$-ray computed tomography. Soil Sci. Soc. Am. J. 60, 1718-1724 